$\xi=-1$

\title{
Performance analysis of direct detection optical OFDM systems
}

\author{
Kumar D, Anilkumar $\mathbf{R}^{1 *}$, Vijayakumar $\mathrm{C} \mathrm{N}^{2}$, S Bhargavi ${ }^{3}$ \\ ${ }^{1}$ Asst. Professor, Dept. of TCE S J C Institute of Technology Chickballapur, India \\ ${ }^{2}$ Professor, Dept. of ECE E W Institute of Technology, Bangalore, India \\ ${ }^{3}$ HOD, Dept. of TCE S J C Institute of Technology, Chickballapur, India \\ *Corresponding author E-mail: kumard243@gmail.com
}

\begin{abstract}
Orthogonal frequency division multiplexing (OFDM) is a multicarrier modulation technique, in which the data information is carried over many lower rate subcarriers. This technique is started effectively using in both line communication and wireless communication systems. This modulation technique has been actively started in the field of light wave communication called optical OFDM (OOFDM) system. OOFDM is a multicarrier modulation technique; it is used to overcome the problem of inter-symbol interference due to the chromatic dispersion and polarization mode dispersion of the fiber channel. In this paper, we have done simulation on direct detection optical OFDM (DDOOFDM) system with data rate of 10Gbps and measuring performance analysis of DDOOFDM system with respect to the received optical power and various full width half maximum value of the continuous wave laser source. This analysis is simulated using RSOFT Design Group OptSim Version-5.2.
\end{abstract}

Keywords: Coherent Detection Optical OFDM (COOFDM); Direct Detection Optical OFDM (DDOOFDM); Full Width Half Maximum (FWHM); MachZehnder Modulator (MZM); Optical Band Pass Filter (OBPF); Optical Single Sideband (OSSB).

\section{Introduction}

In recent years fiber channel is a most wanted line communication because of its high bandwidth in the order of $10^{14} \mathrm{~Hz}$ and low bit error rate in the order of $10^{-9}$ without any coding technique. It is suffering two major problems one is attenuation and other is dispersion. Attenuation can be eliminated using erbium doped fiber amplifier. Orthogonal frequency division multiplexing (OFDM) is most preferable modulation technique to overcome the problem of dispersion of the channel. OFDM converts the high data rate of information to many lower data rate of subcarriers [1]. The advancement in the digital signal processing (DSP) technology, OFDM modulation technique effectively started in optical fiber channel and free space optics. Applications of OFDM technique are used in Wi-Fi (IEEE 802.11), WiMAX (IEEE 802.16), fourthgeneration mobile communication technology and digital audio or video broadcasting systems (DAB/DVB). In recent years we observe the drastic increase of optical OFDM (OOFDM) in the field of fiber communication [8]. This is better modulation technique for long-haul transmission of coherent detection optical OFDM system or direct detection of optical OFDM system [9], [10].

According to the different receiver structure, OOFDM is classified in to two types one is direct detection optical OFDM (DDOOFDM) system and other is coherent optical OFDM (COOFDM) system. DDOOFDM system was first introduced by Lowery in 2006 [2]. Similarly COOFDM system which can eliminate the chromatic dispersion was also investigated in 2006 by Shieh [3]. There are some differences between the DDOOFDM system and COOFDM system. DDOOFDM system is effectively used for short distance communication and design is very simple and less expense compare to the COOFDM system. DDOOFDM system requires few components at the transmitter and receiver side section. It's required spectral gap after optical modulation to eliminate the inter-modulations products in the photodiode at the receiver side [6]. Therefore, it needs more bandwidth for transmission and decreases the spectral efficiency. But it is used to measure only the amplitude of the optical signal. COOFDM system is best suitable for long haul transmission with high data rate [5]. To achieve high performance of the COOFDM system, it requires a very narrow line width of same laser source at the transmitter and receiver end of the COOFDM system [12]. Laser source and local oscillator laser source have very narrow line width and effective digital signal processing (DSP) components are essential for COOFDM system. The receiver design is very complex in COOFDM system compare to the DDOOFDM system because phase and frequency synchronization at receiver is very complex and it require very efficient digital signal processing components [13]. Therefore, COOFDM system is more expensive than the DDOOFDM system. DDOOFDM system gives, simple receiver structure which contains only one photo diode and gives decent performance compare to the COOFDM system. [15] Discussed about a project, in this project an automatic meter reading system is designed using GSM Technology. The embedded micro controller is interfaced with the GSM Module. This setup is fitted in home. The energy meter is attached to the micro controller. This controller reads the data from the meter output and transfers that data to GSM Module through the serial port. The embedded micro controller has the knowledge of sending message to the system through the GSM module. Another system is placed in EB office, which is the authority office. When they send "unit request" to the microcontroller which is placed in home. Then the unit value is sent to the EB office PC through GSM module. According to the readings, the authority officer will send the information about the bill to the customer. If the customer doesn't pay bill on-time, the power supply to the corresponding home power unit is cut, by sending the command through to the microcontroller. Once the payment of bill is done the power supply is given to the customer. 
Power management concept is introduced, in which during the restriction mode only limited amount of power supply can be used by the customer.

In this work, we have simulated 10Gbps DDOOFDM system with $50 \mathrm{~km}$ standard single mode fiber channel and measure the performance analysis of DDOOFDM system with respect to received optical power. Full results of simulated $10 \mathrm{Gbps}$ data transmission are reported in this paper. In this work we have explained DDOOFDM system in section II, simulation steps and corresponding simulations results are discussed in section III. Finally we discussed conclusion in section IV.

\section{Architecture of DDOOFDM system}

\subsection{Architecture design}

DDOOFDM system is suitable for short distance and less expensive applications. Its architecture design is less complex than the COOFDM system. But it gives the less spectral efficiency compare to the COOFDM system. The schematic representation of the DDOOFDM system is shown in Fig.1.

Baseband OFDM Generation

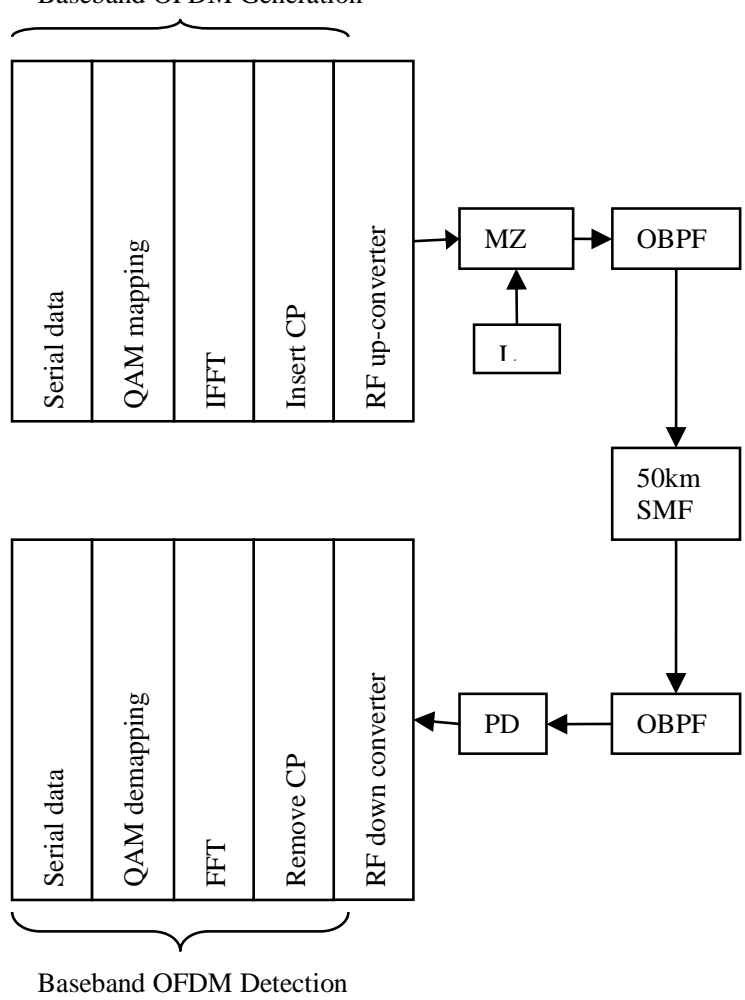

Fig. 1: Architecture of DDOOFDM System.

The high input serial data rate are parallelized using serial to parallel converter. The parallel blocks of data are applied to QAM modulator to mapping the signal in to complex form. In our simulation we have used 16-QAM modulation and demodulation. After QAM modulation both in phase and qudrarure phase components applied to the IFFT to get the time domain OFDM signals. The size of IFFT represents the number of subcarriers; we used 128 subcarriers in our simulation. Zero padding is also required in OFDM to avoid the aliasing at the time of digital to analog conversion. The range of IFFT usually lies between the 128 and 1024 . Now each OFDM symbol has duration of $T_{s}=N / R$, here $N$ is the number of subcarriers used in IFFT and R is the data rate of input signal. This reduces the dispersion effect in the fiber channel. To reduce the inter-symbol interference between the OFDM symbols, a guard band or guard time is required; this guard time is called the cyclic prefix. It is defined as copying the last $1 / 4$ portion of
OFDM symbol to beginning of same OFDM symbol. After cyclic prefix addition, time domain OFDM signal is serialized and converted to analog form using serial to parallel and digital to analog converters. After that baseband OFDM signal is up-converted using RF intermediate frequency and applied to Mach-Zehnder modulator (MZM) with continuous wave laser source to modulate RF OFDM signal to optical OFDM signal [16]. Here MZM operation should be in linear region and laser source have very low full width half maximum value in the order of $\mathrm{kHz}$. After MZM modulation OOFDM signal is applied to optical band pass filter to eliminate the lower side bands and makes it become the single sided optical OFDM signal. Single mode fiber is used to transmit the single sided optical OFDM signal with attenuation and dispersion constant. We have used $50 \mathrm{~km}$ standard single mode fiber with $0.2 \mathrm{~dB} / \mathrm{km}$ attenuation and $16 \mathrm{ps} / \mathrm{nm} / \mathrm{km}$ dispersion value. After $50 \mathrm{~km}$ fiber we used PIN photo diode at receiver to convert optical OFDM signal to RF OFDM signal with responsivity of $0.9 \mathrm{~A} / \mathrm{W}$ and dark current of $0.1 \mathrm{nA}$. For down conversion RF OFDM signal is applied to RF down converter with local oscillator having same inter mediate frequency. The down converted OFDM signal again converted to digital form and parallel blocks data using analog to digital converter and serial to parallel converter. Cyclic prefix is removed from each OFDM symbol and applied to FFT to get back the frequency domain parallel QAM symbols. Each QAM symbols are demodulated using QAM demodulator. After QAM demodulator the parallel information bits are serialized using serial to parallel converter. [4] proposed a novel method for secure transportation of railway systems has been proposed in this project. In existing methods, most of the methods are manual resulting in a lot of human errors. This project proposes a system which can be controlled automatically without any outside help. This project has a model concerning two train sections and a gate section. The railway sections are used to show the movement of trains and a gate section is used to show the happenings in the railway crossings. The scope of this project is to monitor the train sections to prevent collisions between two trains or between humans and trains and to avoid accidents in the railway crossings. Also an additional approach towards effective power utilization has been discussed. Five topics are discussed in this project : 1) Detection of obstacles in front of the train;2) Detection of cracks and movements in the tracks;3) Detection of human presence inside the train and controlling the electrical devices accordingly 4) Updating the location of train and sharing it with other trains automatically 5) Controlling the gate section during railway crossing. This project can be used to avoid accidents in the railway tracks.

\subsection{Mathematical concepts}

Optical OFDM is the best solution to eliminate the dispersion in fiber channel and it is not required dispersion compensation fiber. First we represent mathematically time domain OFDM signal in (1)

$$
\mathrm{S}_{1}(\mathrm{t})=\sum_{\mathrm{k}=1}^{\mathrm{N}}\left(\mathrm{a}_{\mathrm{k}} \cos \mathrm{k} \Omega \mathrm{t}+\mathrm{b}_{\mathrm{k}} \sin \mathrm{k} \Omega \mathrm{t}\right)
$$

From (1), $\Omega$ represents fundamental frequency of the subcarriers, $\mathrm{k} \Omega$ represents $\mathrm{k}^{\text {th }}$ subcarrier OFDM signal frequency, $\mathrm{a}_{\mathrm{k}}$ represents the in phase component and $b_{k}$ represents the quadrature phase component of the complex symbol in $\mathrm{k}^{\text {th }}$ subcarrier and $\mathrm{N}$ represents the number of subcarriers used in IFFT block [11]. After digital to analog conversion the signal is degraded because of effect of digital signal processing components [11]. We define the degraded coefficient of $\mathrm{k}^{\text {th }}$ subcarrier as $\mathrm{X}_{\mathrm{k}}$, the OFDM signal after modulation is defined in (2)

$\mathrm{S}_{2}(\mathrm{t})=\sum_{\mathrm{k}=1}^{\mathrm{N}} \mathrm{X}_{\mathrm{k}}\left(\mathrm{a}_{\mathrm{k}} \cos \mathrm{k} \Omega \mathrm{t}+\mathrm{b}_{\mathrm{k}} \sin \mathrm{k} \Omega \mathrm{t}\right)$

We have assumed the monochromatic laser source having a frequency of $\omega_{0}$ and amplitude of optical signal is $A_{0}$, then the optical signal is defined as 
$C(t)=A_{0} \cos \left(\omega_{0} t\right)$

The electrical OFDM signal is applied to MZM intensitymodulator with continuous wave laser source, it converts electrical OFDM signal to optical OFDM signal. In MZM we have to maintain linear operating voltage [11]. The optical OFDM signal after the MZM intensity modulator is defined as

$\mathrm{E}_{\text {out }}(\mathrm{t})=\mathrm{A}_{0} \cos \left(\omega_{0} \mathrm{t}\right) \times\left[1+\gamma \sum_{\mathrm{k}=1}^{\mathrm{N}} \mathrm{X}_{\mathrm{k}}\left(\mathrm{a}_{\mathrm{k}} \cos \mathrm{k} \Omega \mathrm{t}+\right.\right.$ $\left.\mathrm{b}_{\mathrm{k}} \sin \mathrm{k} \Omega \mathrm{t}\right)$ ]

From (4), $\gamma$ represents the optical modulation index of the MZM at linear region [11]. After MZM the optical OFDM signal propagation over L-km length of the fiber channel. We considered the propagation constant of fiber $\beta(\omega)$, attenuation constant $\alpha(\mathrm{L})$ and define optical OFDM signal transmission through L-km length of fiber as

$$
\begin{aligned}
& \mathrm{E}_{\text {out }}(\mathrm{t})=\mathrm{A}_{0} \gamma \alpha(\mathrm{L}) \sum_{\mathrm{k}=1}^{\mathrm{N}} \mathrm{X}_{\mathrm{k}} \cos \times\left(\omega_{0} \mathrm{t}-\right. \\
& \left.\frac{\beta\left(\omega_{0}+\mathrm{k} \Omega\right)+\beta\left(\omega_{0}-\mathrm{k} \Omega\right)}{2} \mathrm{~L}\right) \times\left[\mathrm{a}_{\mathrm{k}} \cos \left(\mathrm{k} \Omega \mathrm{t}-\frac{\beta\left(\omega_{0}+\mathrm{k} \Omega\right)-\beta\left(\omega_{0}-\mathrm{k} \Omega\right)}{2} \mathrm{~L}\right) \times\right. \\
& \left.\mathrm{b}_{\mathrm{k}} \sin \left(\mathrm{k} \Omega \mathrm{t}-\frac{\beta\left(\omega_{0}+\mathrm{k} \Omega\right)-\beta\left(\omega_{0}-\mathrm{k} \Omega\right)}{2} \mathrm{~L}\right)\right]+\mathrm{A}_{0} \alpha(\mathrm{L}) \cos \left(\omega_{0} \mathrm{t}-\right. \\
& \left.\beta\left(\omega_{0}\right) \mathrm{L}\right)
\end{aligned}
$$

In(5), we neglect the fiber nonlinearity and noise terms of the fiber channel. For simplifying (5), we consider constants $\beta_{1}, \beta_{2}$ and $\beta_{3}$. These constants terms are defined as, $\beta_{1}=$ $\beta\left(\omega_{0}+\mathrm{k} \Omega\right), \beta_{2}=\beta\left(\omega_{0}-\mathrm{k} \Omega\right), \beta_{3}=\beta\left(\omega_{0}\right)$ and substitute these values in (5), then it becomes

$$
\begin{aligned}
& \mathrm{E}_{\text {out }}(\mathrm{t})=\mathrm{A}_{0} \gamma \alpha(\mathrm{L}) \sum_{\mathrm{k}=1}^{\mathrm{N}} \mathrm{X}_{\mathrm{k}} \cos \left(\omega_{0} \mathrm{t}-\frac{\left(\beta_{1}+\beta_{2}\right)}{2} \mathrm{~L}\right) \times \\
& {\left[\mathrm{a}_{\mathrm{k}} \cos \left(\mathrm{k} \Omega \mathrm{t}-\frac{\left(\beta_{1}-\beta_{2}\right)}{2} \mathrm{~L}\right)+\mathrm{b}_{\mathrm{k}} \sin \left(\mathrm{k} \Omega \mathrm{t}-\frac{\left(\beta_{1}-\beta_{2}\right)}{2} \mathrm{~L}\right)\right]+} \\
& \mathrm{A}_{0} \alpha(\mathrm{L}) \cos \left(\omega_{0} \mathrm{t}-\beta_{3} \mathrm{~L}\right)
\end{aligned}
$$

In order to simplify (6) we assume

$\mathrm{Q}_{\mathrm{k}}=\mathrm{X}_{\mathrm{k}}\left[\mathrm{a}_{\mathrm{k}} \cos \left(\mathrm{k} \Omega \mathrm{t}-\frac{\left(\beta_{1}-\beta_{2}\right)}{2} \mathrm{~L}\right)+\mathrm{b}_{\mathrm{k}} \sin \left(\mathrm{k} \Omega \mathrm{t}-\frac{\left(\beta_{1}-\beta_{2}\right)}{2} \mathrm{~L}\right)\right]$

We can write (6) as

$\mathrm{E}_{\text {out }}(\mathrm{t})=\mathrm{A}_{0} \gamma \alpha(\mathrm{L}) \sum_{\mathrm{k}=1}^{\mathrm{N}} \cos \left(\omega_{0} \mathrm{t}-\frac{\left(\beta_{1}+\beta_{2}\right)}{2} \mathrm{~L}\right) \times \mathrm{Q}_{\mathrm{k}}+$

$A_{0} \alpha(L) \cos \left(\omega_{0} t-\beta_{3} L\right)$

At the receiver side we have used PIN photo diode to convert optical OFDM signal to RF OFDM signal [11]. Then we define the unfiltered photon electrical current from PIN photodiode as

$$
\begin{aligned}
& I \propto\left|E_{\text {out }}(t)\right|^{2} \\
& I=\mu\left|E_{\text {out }}(t)\right|^{2}
\end{aligned}
$$

Where $\mu$ is a constant, it is consider to be 1 [11], substitute (7) in (8), then the expression for photo electrical current becomes

$$
\begin{aligned}
& \mathrm{I}=\left(\mathrm{A}_{0} \gamma \alpha(\mathrm{L}) \sum_{\mathrm{k}=1}^{\mathrm{N}} \cos \left(\omega_{0} \mathrm{t}-\frac{\left(\beta_{1}+\beta_{2}\right)}{2} \mathrm{~L}\right) \times \mathrm{Q}_{\mathrm{k}}\right)^{2}+ \\
& 2 \gamma \mathrm{A}_{0}^{2} \alpha^{2}(\mathrm{~L}) \cos \left(\omega_{0} \mathrm{t}-\beta_{3} \mathrm{~L}\right) \times\left(\sum_{\mathrm{k}=1}^{\mathrm{N}} \cos \left(\omega_{0} \mathrm{t}-\frac{\left(\beta_{1}+\beta_{2}\right)}{2} \mathrm{~L}\right) \times\right. \\
& \left.\mathrm{Q}_{\mathrm{k}}\right)+\mathrm{A}_{0}^{2} \alpha^{2}(\mathrm{~L}) \cos ^{2}\left(\omega_{0} \mathrm{t}-\beta_{3} \mathrm{~L}\right)
\end{aligned}
$$

$$
\begin{aligned}
& \mathrm{I}=\gamma^{2} \mathrm{~A}_{0}^{2} \alpha^{2}(\mathrm{~L})\left[\sum_{\mathrm{k}=1}^{\mathrm{N}} \cos \left(\omega_{0} \mathrm{t}-\frac{\left(\beta_{1}+\beta_{2}\right)}{2} \mathrm{~L}\right) \times \mathrm{Q}_{\mathrm{k}}\right]^{2}+ \\
& \gamma \mathrm{A}_{0}^{2} \alpha^{2}(\mathrm{~L})\left[\sum_{\mathrm{k}=1}^{\mathrm{N}} \cos \left(2 \omega_{0} \mathrm{t}-\frac{\left(\beta_{1}+\beta_{2}+2 \beta_{3}\right)}{2} \mathrm{~L}\right) \times\right. \\
& \left.\mathrm{Q}_{\mathrm{k}}+\sum_{\mathrm{k}=1}^{\mathrm{N}} \cos \left(\frac{\beta_{1}+\beta_{2}-2 \beta_{3}}{2} \mathrm{~L}\right) \times \mathrm{Q}_{\mathrm{k}}\right]^{2} \\
& +\mathrm{A}_{0}^{2} \alpha^{2}(\mathrm{~L}) \cos ^{2}\left(\omega_{0} \mathrm{t}-\beta_{3} \mathrm{~L}\right)
\end{aligned}
$$

We can write (10) as

$$
\begin{aligned}
& I=\gamma^{2} A_{0}^{2} \alpha^{2}(L)\left[\sum_{\mathrm{k}=1}^{\mathrm{N}} \mathrm{M} \times \mathrm{Q}_{\mathrm{k}}\right]^{2}+\gamma \mathrm{A}_{0}^{2} \alpha^{2}(\mathrm{~L})\left[\sum_{\mathrm{k}=1}^{\mathrm{N}} \mathrm{P} \times \mathrm{Q}_{\mathrm{k}}+\right. \\
& \left.\sum_{\mathrm{k}=1}^{\mathrm{N}} \cos \left(\frac{\beta_{1}+\beta_{2}-2 \beta_{3}}{2} \mathrm{~L}\right) \times \mathrm{Q}_{\mathrm{k}}\right]+\mathrm{A}_{0}^{2} \alpha^{2}(\mathrm{~L}) \mathrm{R}^{2}
\end{aligned}
$$

Where $\mathrm{M}, \mathrm{P}$ and $\mathrm{R}$ denote the light wave terms, optical carrier signal contains all these terms [11]. These terms are individually defined as

$$
\begin{aligned}
& M=\cos \left(\omega_{0} t-\frac{\left(\beta_{1}+\beta_{2}\right)}{2} L\right) \\
& P=\cos \left(2 \omega_{0} t-\frac{\left(\beta_{1}+\beta_{2}+2 \beta_{3}\right)}{2} L\right) \\
& R=\cos \left(\omega_{0} t-\beta_{3} L\right)
\end{aligned}
$$

The light wave terms M, P and R are considered being DC components. Finally we get the directly detected OFDM signal from (10) as

$$
\begin{aligned}
& \mathrm{I}=\gamma^{2} \mathrm{~A}_{0}^{2} \alpha^{2}(\mathrm{~L})\left[\sum_{\mathrm{k}=1}^{\mathrm{N}} \mathrm{Q}_{\mathrm{k}}\right]^{2}+\gamma \mathrm{A}_{0}^{2} \alpha^{2}(\mathrm{~L})\left[\sum_{\mathrm{k}=1}^{\mathrm{N}} \cos \left(\frac{\beta_{1}+\beta_{2}-2 \beta_{3}}{2} \mathrm{~L}\right) \times\right. \\
& \left.\mathrm{Q}_{\mathrm{k}}+1\right]+\mathrm{A}_{0}^{2} \alpha^{2}(\mathrm{~L})
\end{aligned}
$$

We can write (12) as

$$
\begin{aligned}
& \mathrm{I}=\gamma \mathrm{A}_{0}^{2} \alpha^{2}(\mathrm{~L})\left[\sum_{\mathrm{k}=1}^{\mathrm{N}} \cos \left(\frac{\beta_{1}+\beta_{2}-2 \beta_{3}}{2} \mathrm{~L}\right) \times \mathrm{Q}_{\mathrm{k}}\right]+ \\
& \gamma^{2} \mathrm{~A}_{0}^{2} \alpha^{2}(\mathrm{~L})\left[\sum_{\mathrm{k}=1}^{\mathrm{N}} \mathrm{Q}_{\mathrm{k}}\right]^{2}+(1+\gamma) \mathrm{A}_{0}^{2} \alpha^{2}(\mathrm{~L})
\end{aligned}
$$

By observing (13), first, second and third terms gives the RF OFDM signal, nonlinearity of the signal and DC component of the signal respectively. The DC component is removed using filters. The nonlinearity term can be removed using two methods, one is sufficient allocation of the guard band and other method is preemphasis technique [11], [18].

\section{Simulation setup}

DDOOFDM system simulation model is developed in the simulation tool RSOFT Design Group OptSim Version-5.2. A 10Gbps digital data stream is converted to parallel blocks of data then applied 16-QAM to map the signal in complex constellation points. The output of QAM modulator gives in-phase and qudrature phase component with each bandwidth of $2.5 \mathrm{GHz}$. After QAM modulation the signal is passed to IFFT block to generate the time domain RF OFDM signal. IFFT block have 128 subcarriers. The cyclic prefix is added after the IFFT block with a $1 / 4^{\text {th }}$ of OFDM symbol duration. In Fig. 2 we have showed spectrum of RF OFDM signal with an OFDM bandwidth of $2.5 \mathrm{GHz}$.

We can write (9) as 


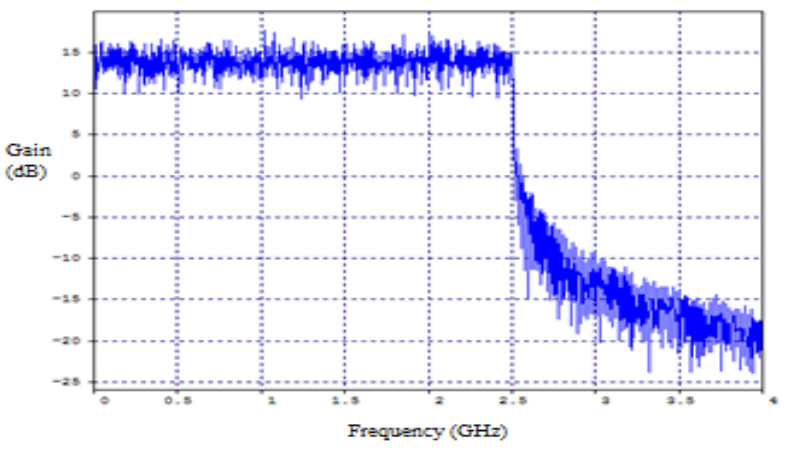

Fig. 2: Spectrum of the RF OFDM Signal after IFFT.

After that RF OFDM signal is serialized and converted to analog form using serial to parallel and digital to analog converters. The base band RF OFDM signal is up-converted using RF upconversion with local oscillator frequency of $6 \mathrm{GHz}$. After upconversion the RF OFDM signal has a bandwidth of $5 \mathrm{GHz}$ and spectral gap is $3.5 \mathrm{GHz}$. The total bandwidth of RF up-converted RF OFDM signal is $8.5 \mathrm{GHz}$ and it is shown in Fig. 3.

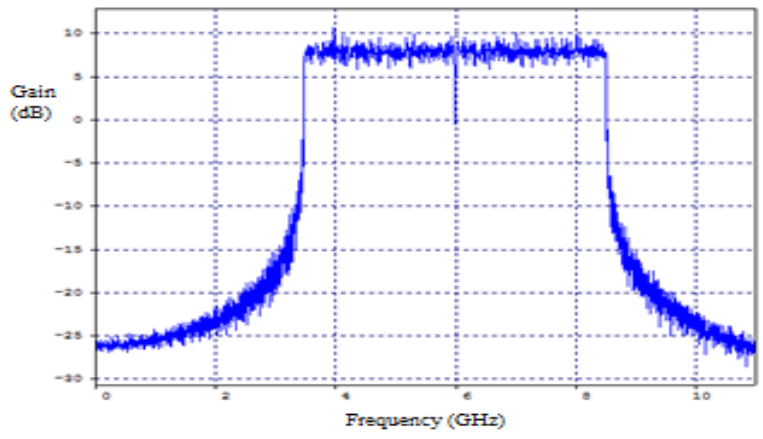

Fig. 3: Spectrum of the RF Up-Converted OFDM Signal.

Up-converted RF OFDM signal is applied to MZM with continuous wave laser source having optical carrier signal at $1550 \mathrm{~nm}$ wavelength. We applied 2V bias voltage to MZM and we maintained MZM operation in linear region. We have maintained continuous wave laser source with a full width half maximum value of $1 \mathrm{MHz}$, operating wavelength of $1550 \mathrm{~nm}$ and optical power of $10 \mathrm{dBm}$. The optical spectrum of the continuous wave laser source is shown in Fig. 4. The optical spectrum is maximum and very narrow at the frequency of $193.414 \mathrm{THz}$.

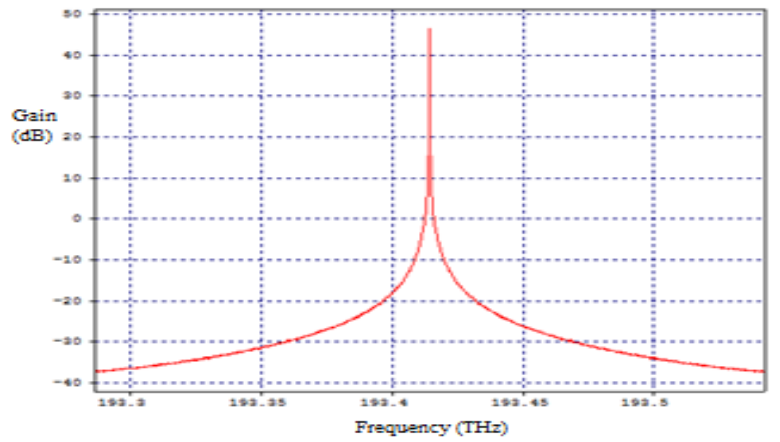

Fig. 4: Optical Spectrum of the Continuous Wave Laser Source.

The spectrum of the optical modulated OFDM signal after MZM is shown Fig. 5

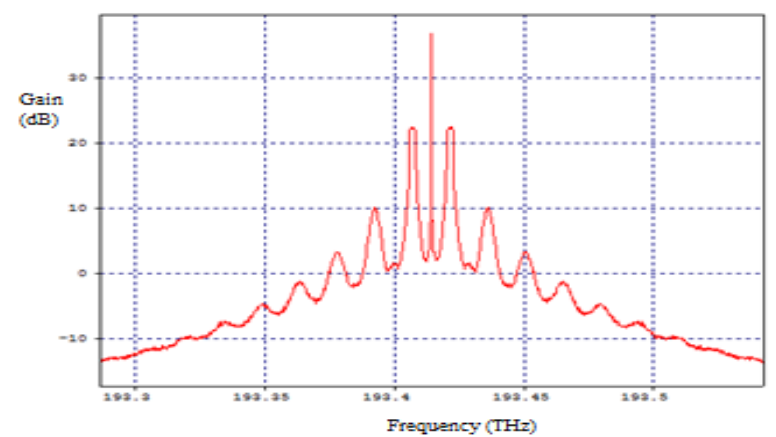

Fig. 5: Optical Spectrum of the OOFDM Signal after MZM.

Fig. 5 shows double sided optical OFDM signal with total bandwidth of $17 \mathrm{GHz}$ and also contains lower side bands of optical signal. The use of raised cosine optical band pass filter makes double sided OOFDM signal to single sided OOFDM signal, saves the bandwidth and it also eliminate other lower side bands. The spectrum of the single sided OOFDM signal after the optical band pass filter (OBPF) is shown in Fig. 6.

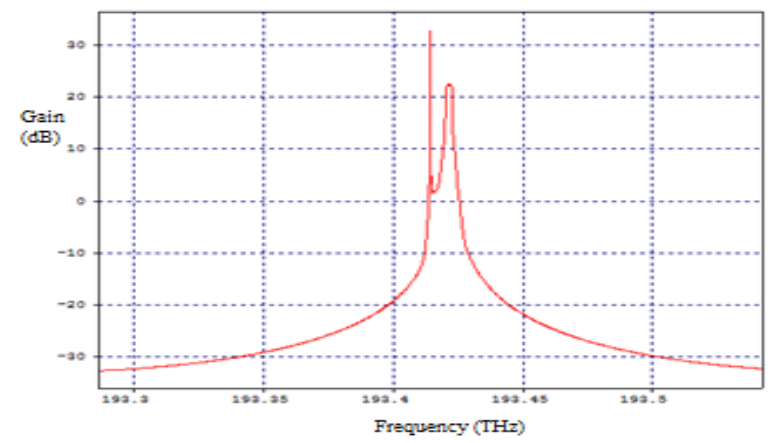

Fig. 6: Optical Spectrum of the Single Sided OOFDM Signal after OBPF.

$50 \mathrm{~km}$ standard single mode fiber (SSMF) used to transmit single sided OOFDM signal with a $0.2 \mathrm{~dB} / \mathrm{km}$ attenuation loss and $16 \mathrm{ps} / \mathrm{nm} / \mathrm{km}$ dispersion value. After $50 \mathrm{~km}$ SSMF we are using PIN photo diode at receiver side to convert OOFDM signal to RF OFDM signal with reponsivity of $0.9 \mathrm{~A} / \mathrm{W}$ and dark current of 0.1nA. Fig. 7 shows the optical to electrical down converted spectrum of the RF OFDM signal after PIN photo diode.

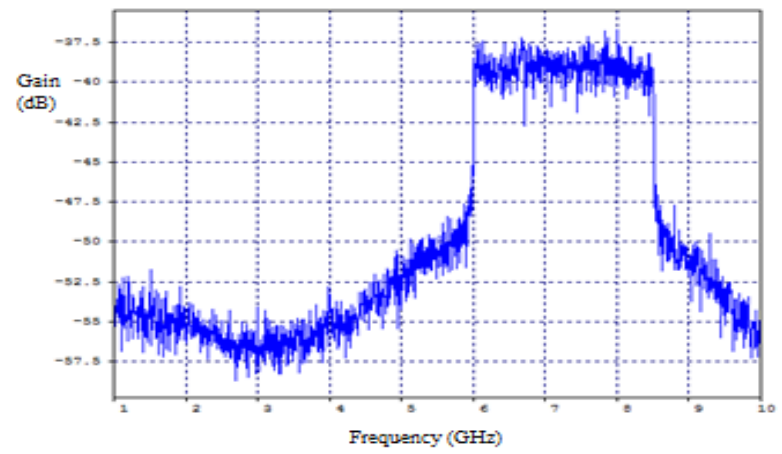

Fig. 7: Spectrum of the RF OFDM Signal after the PIN Diode.

From Fig. 7 we can observe, spectral gap contains intermodulation products and noise from the photodiode and fiber. These terms easily eliminated using filters. The directly detected RF OFDM signal is applied to amplifier with a gain of $15 \mathrm{~dB}$ and passed through RF down converter with local oscillator intermediate frequency of $6 \mathrm{GHz}$ to recover the in-phase and qudrature phase components of the signals. The down converted OFDM signal again converted to digital form and parallel blocks data using analog to digital converter and serial to parallel converter. Fig. 8 shows the spectrum of the RF OFDM signal after down conversion. 


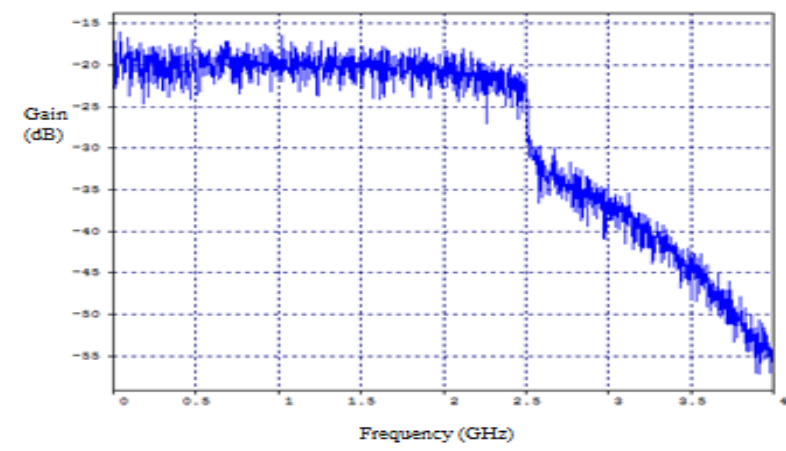

Fig. 8: Spectrum of RF OFDM Signal after Down Convertor.

Fig. 8 shows the in-phase component of OFDM signal having bandwidth of $2.5 \mathrm{GHz}$. After getting the in-phase and qudrature phase signals, the cyclic prefix is removed from each OFDM block and applied to FFT block with 128 subcarriers. After FFT block we get the frequency domain QAM symbols from OFDM signal. After that each QAM symbol is demodulated using 16QAM demodulator. The QAM demodulator produces the parallel blocks of data. This data can be serialized with the help of parallel to serial converter. The performance of $10 \mathrm{Gbps}$ direct detection optical OFDM system with $10 \mathrm{~km}$ and $50 \mathrm{~km}$ length of SSMF channel is shown in Fig. 9.

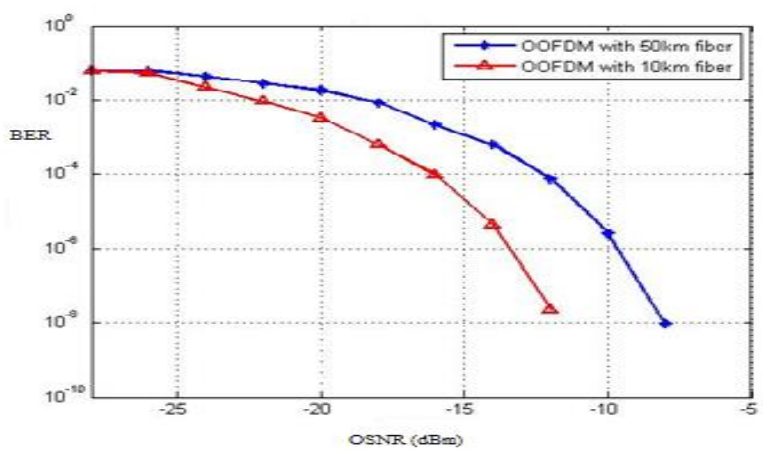

Fig. 9: Performance of 10Gbps DDOOFDM System with $10 \mathrm{~km}$ and $50 \mathrm{~km}$ Length of SSMF Channel.

We have plotted the graph of BER vs OSNR for both $50 \mathrm{~km}$ and $10 \mathrm{~km}$ length of the SSMF channel. In this simulation we considered continuous wave laser source with full width half maximum (FWHM) value of $1 \mathrm{MHz}$ and optical power of $10 \mathrm{dBm}$. From Fig. 9, we observe that for $50 \mathrm{~km}$ length of SSMF channel, at received power level of $-8 \mathrm{dBm}$, the corresponding BER is $10^{-8}$. Similarly for $10 \mathrm{~km}$ length of SSMF channel, at received power level of $12 \mathrm{dBm}$, the corresponding BER is $10^{-8}$. The length of fiber increases the power requirement to transmit the optical OFDM signal also increases. This is due to the attenuation and dispersion of fiber. We can also conclude that attenuation also effects BER of the system. For the long length of fiber we have to use good photo detector and dispersion compensation fiber for better performance of the system [19].

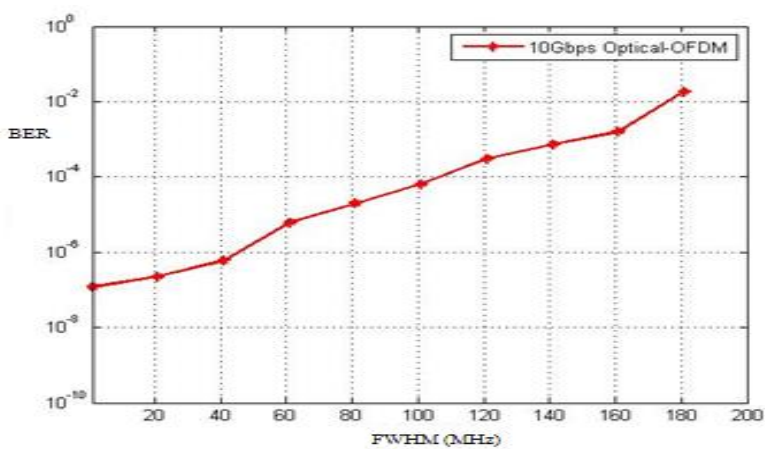

Fig. 10: BER vs. Full Width Half Maximum Value of Laser Source.
Fig. 10 shows the BER performance over varying full width half maximum (FWHM) value of continuous wave laser source with optical power of $10 \mathrm{dBm}$. At FWHM value of $1 \mathrm{MHz}$, the corresponding BER is $10^{-7}$ for direct detection optical OFDM system with $50 \mathrm{~km}$ length of SSMF. Increasing FWHM value of laser source, the BER also increases. From Fig. 10 we observe that, FWHM value of $180 \mathrm{MHz}$, the BER is $10^{-2}$. For better performance of direct detection optical OFDM system we have to maintain FWHM value of laser source is less than $1 \mathrm{MHz}$.

\section{Conclusion}

Optical OFDM is the best solution to overcome the problem of inter-symbol interference due to the dispersion of the optical fiber channel (chromatic dispersion and polarization mode dispersion). Simulation results show that $10 \mathrm{Gbpsdirect}$ detection optical OFDM (DDOOFDM) system is effectively operated over $50 \mathrm{~km}$ length of SSMF channel without dispersion compensation of fiber with OFDM bandwidth of $2.5 \mathrm{GHz}$. For better performance of DDOOFDM system, it is required to maintain laser source FWHM value less than $1 \mathrm{MHz}$. The single sided optical OFDM signal transmission can eliminate the chromatic dispersion in DDOOFDM system. For DDOOFDM system with transmission distance beyond 50km SSMF, dispersion compensation should be necessary.

\section{References}

[1] J. Armstong, "OFDM for optical communications", IEEE journal of Light wave technology, vol. 27, no.3, pp.189-204, 2009 https://doi.org/10.1109/JLT.2008.2010061.

[2] J. Lowery, Liang. Du, and Jean Armstrong, "Orthogonal frequency division multiplexing for adaptive dispersion compensation in long haul WDM systems", in Optical Fiber Communication Conference, PP. 1-3, Optical Society of America, 2006.

[3] W. Sieh and C. Athaudage, "Coherent optical orthogonal frequency division multiplexing", Electronics Letters, vol. 42, no. 10, pp.587589, 2006. https://doi.org/10.1049/el:20060561.

[4] Christo Ananth, K.Nagarajan, Vinod Kumar.V., "A Smart Approach for Secure Control of Railway Transportation Systems", International Journal of Pure and Applied Mathematics, Volume 117 , Issue 15, 2017, (1215-1221).

[5] S. L. Jansen, I. Morita, Tim C. W. Schenk, and H. Tanaka, "Coherent optical 25.8Gbps OFDM transmission over 4160km SSMF", IEEE Journal of Light Wave Technology, vol. 26, no. 1, pp. 6-15, 2008. https://doi.org/10.1109/JLT.2007.911888.

[6] L. Mehedy, M. Bakul, and A. Nirmalathas, "Single channel directly detected optical OFDM towards higher spectral efficiency and simplicity in 100Gbps Ethernet and beyond", Opt. Commun. Netw.vol 3, no. 5 MAY 2011.

[7] Shu-Hao Fan, Jianijun Yu, and Gee-kung chang, "Optical OFDM scheme using uniform power transmission to mitigate peak to average power effect over $1040 \mathrm{~km}$ single mode fiber", Opt. Commun. Netw.vol. 2, no. 9, pp. 711-715, September 2010 https://doi.org/10.1364/JOCN.2.000711.

[8] Neda Cvijetic, "OFDM for next generation optical access networks", IEEE Journal of Light Wave Technology, vol. 30, no. 4, pp. 493-503, February 15, 2012 https://doi.org/10.1109/JLT.2011.2173905.

[9] Zizheng Cao, Jianjun Yu, Wenpei Wang, Lin Chen, and Ze Dong, "Direct detection optical OFDM transmission system without frequency guard band", IEEE PhotonicsTechnology Letters, vol. 22 no. $11, \quad$ pp. 736-738, June $1, \quad 2010$. https://doi.org/10.1109/LPT.2010.2044656.

[10] Yan Tang, William Shieh, Xingwen Yi and Rob Evans, "Optimum design for RF to optical up convertor in coherent optical OFDM systems", IEEEPhotonics Technology Letters, vol. 19, no. 7, pp. 483-485, April 1, 2007. https://doi.org/10.1109/LPT.2007.893582.

[11] Yang Gao, Jianjun Yu, Jiangnan Xiao, Zizheng Cao, Fan Li, and Lin Chen, "Direct detection optical OFDM transmission system with pre emphasis technique", IEEE Journal of Light Wave Technology,vol. 29, no. 14, pp. 2138-2145, July 15, 2011. https://doi.org/10.1109/JLT.2011.2154299.

[12] W. Shieh, X. Yi, and Y. Tang, "Transmission experiment of multigiga bit coherent optical OFDM syetem over 1000km SSMF fiber", 
Electronics Letters, vol.43, no.3, pp.183-184,2007. https://doi.org/10.1049/el:20073496.

[13] Qi Yang, Yan Tang, Yiran Ma, and William Shieh, "Experimental demonstration and numerical solution of $107 \mathrm{Gbps}$ high spectral efficiency of coherent optical OFDM system", IEEE Journal of Light Wave Technology, vol.27, no.3, pp168-176, 2009. https://doi.org/10.1109/JLT.2008.2007134.

[14] Al Amin, H Takahashi, I. Morita, and H. Tanaka, "100Gbps direct detection optical OFDM transmission on independent polarization tributaries", IEEE Photon.Technol.Letter, vol.22, no.27, pp.468470, 2010. https://doi.org/10.1109/LPT.2010.2040994.

[15] Christo Ananth, Kanthimathi, Krishnammal, Jeyabala, Jothi Monika, Muthu Veni, "GSM Based Automatic Electricity Billing System", International Journal Of Advanced Research Trends In Engineering And Technology (IJARTET), Volume 2, Issue 7, July 2015, pp:16-21.

[16] L. N. Binh, Digital optical communications. CRC press Taylor and Francisgroup, 2009

[17] S. Brendon J, Arthur J. Lowery, and J. Armstrong, "Experimental demonstration of 20Gbps direct detection optical OFDM and $12 \mathrm{Gbps}$ with a colourless transmitter", National Fiber Optics Engineers Conference, Optical Society of America, 2007.

[18] William Shieh and Ivan Djordjevic, OFDM for optical communications. USA, Elsevier, 2010.

[19] John M. Senior, Optical fiber communications. Third edition, Pearson, 2010. 